\title{
Distributed subwavelength grating demultiplexer in SOI
}

\author{
Eric Bisaillon*a, Dawn T. H. Tan ${ }^{\mathrm{b}}$, Marie-Claude Nadeau ${ }^{\mathrm{a}}$, Lukas Chrostowski ${ }^{\mathrm{b}}$, Andrew Kirk ${ }^{\mathrm{a}}$ \\ ${ }^{a}$ Electrical and Computer Engineering Dept., McGill Univ.. Montreal, QC, Canada H3A 2A7; \\ ${ }^{\mathrm{b}}$ Electrical and Computer Engineering Dept., Univ. of British Columbia. Vancouver., BC, V6T 1 Z4
}

\begin{abstract}
A shallow-etched, distributed grating is proposed as a wavelength demultiplexer. The distributed grating constitutes a structure with completely decoupled reflective and diffractive properties. The theory behind the layout of the distributed grating is presented. The structure is modeled using RCWA and FDTD. Modeling results predict up to 79\% efficiency over a $60 \mathrm{~nm}$ wavelength range. Device fabrication results are presented. Early optical characterization of a test structure confirms the expected behavior for the device.
\end{abstract}

Keywords: Diffraction gratings, subwavelength, multiplexer, etched grating, waveguide gratings, echelle, e-beam

\section{INTRODUCTION}

Two technologies have been actively pursued for integrated wavelength division multiplexing (WDM) systems: the etched diffraction grating multiplexer (EDG) and the phase array waveguide (AWG) [1]. In a conventional etched grating device, a diffraction grating structure is etched into a slab waveguide. Light propagated in the slab waveguide and reflects off of deeply etched reflective sidewalls. The slide wall has to be etched for total reflection of the light into the slab waveguide. The retro-reflected light is diffracted by the grating periodicity and spatially separated in the slab. A series of output waveguides are positioned to collect the light. In the conventional grating demultiplexer the grating structure is curved to provide refocusing of the output light. The functionality of the structure is illustrated in Fig. 1.

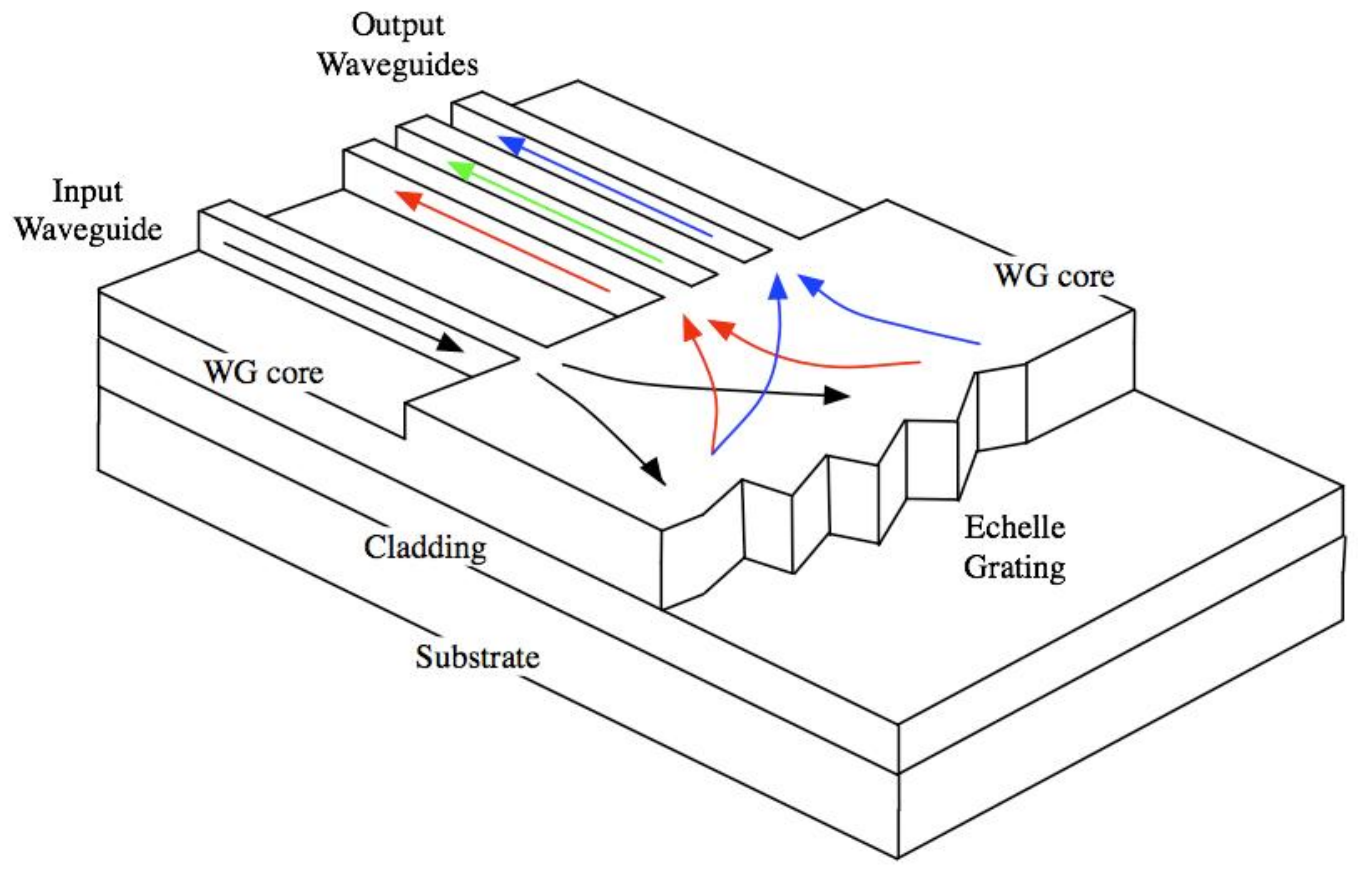

Fig. 1. Layout and operation of an etched grating demultiplexer, showing paths for input and output light.

Integrated Optics: Devices, Materials, and Technologies XI, edited by Yakov Sidorin, Christoph A. Waechter, Proc. of SPIE Vol. 6475, 64750C, (2007) - 0277-786X/07/\$18 - doi: 10.1117/12.700709 
The principal fabrication challenges for EDGs include the requirement for excellent deeply etched sidewall flatness and verticality, necessary to ensure a low reflection loss by the diffraction grating walls [2]. Previously, we proposed a novel approach to the problem of high-reflectivity sidewalls for this type of structure in the form of a shallow-etched, distributed diffraction grating [3]. A distributed reflector replaces the deeply etched grating wall. The diffractive and reflective effects are thus decoupled and can be individually tailored to produce a high efficiency reflective grating [3, 4]. In this talk we present the deisgn and modeling approach for distributed demultiplexers. We begin by describing the layout of the structure, based on a multilayer grating theory, together with the modeling of the structure, using rigorous coupled wave analysis (RCWA) and the Finite-Difference Time-Domain (FDTD) analysis in section 2. In section 3, we discuss the fabrication and early experimental results for the a test structure.

\section{DESIGN}

The layout of a distributed grating is shown in Fig.2. The demultiplexer is built by etching input and output ridge waveguides followed by a free-slab propagation region into the top layer of a silicon-on-insulator (SOI) structure. A shallow depth transmission grating and Bragg region replace the conventional deep-etched grating.

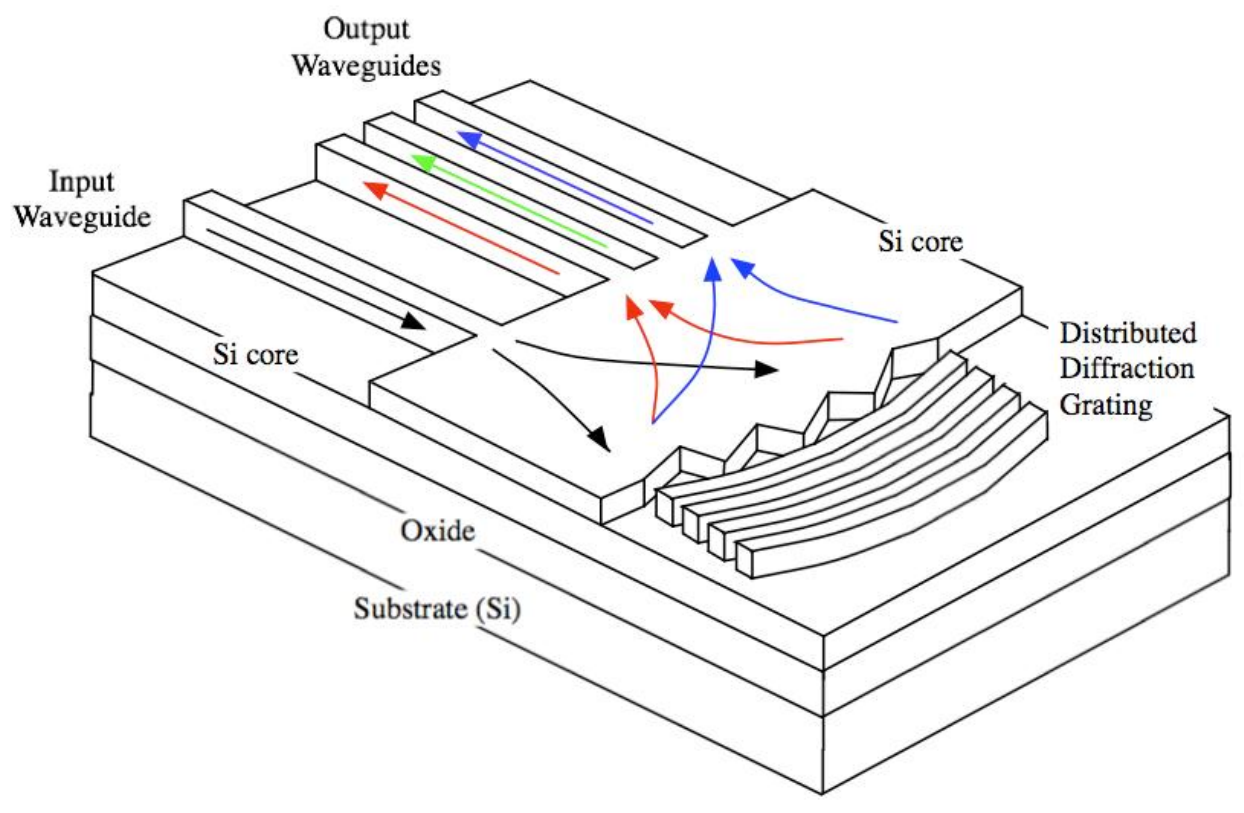

Fig. 2. Distributed EDG Design

The input ridge waveguide was modeled using the 3D beam propagation method (BPM). The effective indices for different slab thicknesses of $\mathrm{Si}$ were used with rigorous coupled-wave analysis (RCWA). The final structure consists of $260 \mathrm{~nm}$ silicon over a $1000 \mathrm{~nm} \mathrm{SiO}_{2}$ cladding. Using the BPM modeling, a $60 \mathrm{~nm}$ etch depth was chosen for the ridge waveguide and distributed regions. For this depth, the 2D (slab) effective indices are approximately 2.272 and 1.728 for the un-etched and etched regions, respectively. The mode propagating in the input waveguide for this structure is shown in Fig. 3 


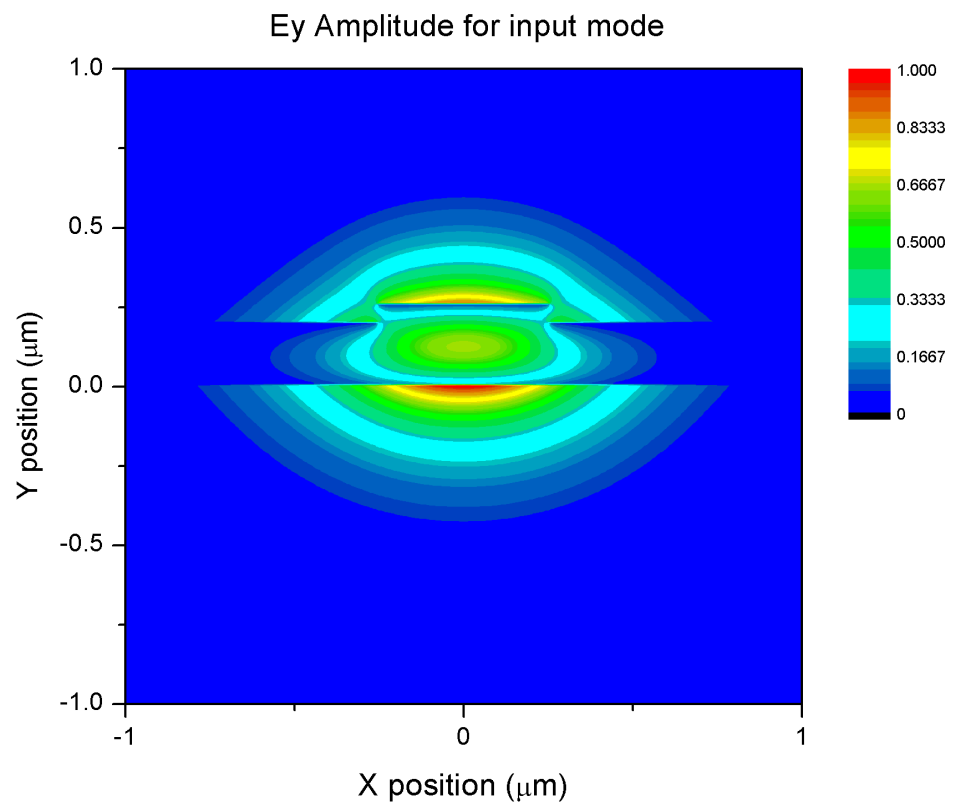

Fig. 3. Input mode profile for test structure waveguide.

The principal advantage of the structure is a single and shallow etch step for the fabrication. The reflective and diffractive properties of the structure can be individually tailored. For example, the Bragg region can be designed to reflect only a portion of the incident spectrum, retro-diffracting only a subset of the input channels for the device.

The light, incident from the single mode input ridge waveguide propagates and expands in the slab region and impinges on the distributed grating. The structure is designed using a modified version of multilayer grating theory [5]. The layout is performed in 2D using the effective indices obtained from BPM. The objective is to layout the multilayer structure for maximum reflection for the diffraction mode of the grating. The layout is shown in Fig. 4. 


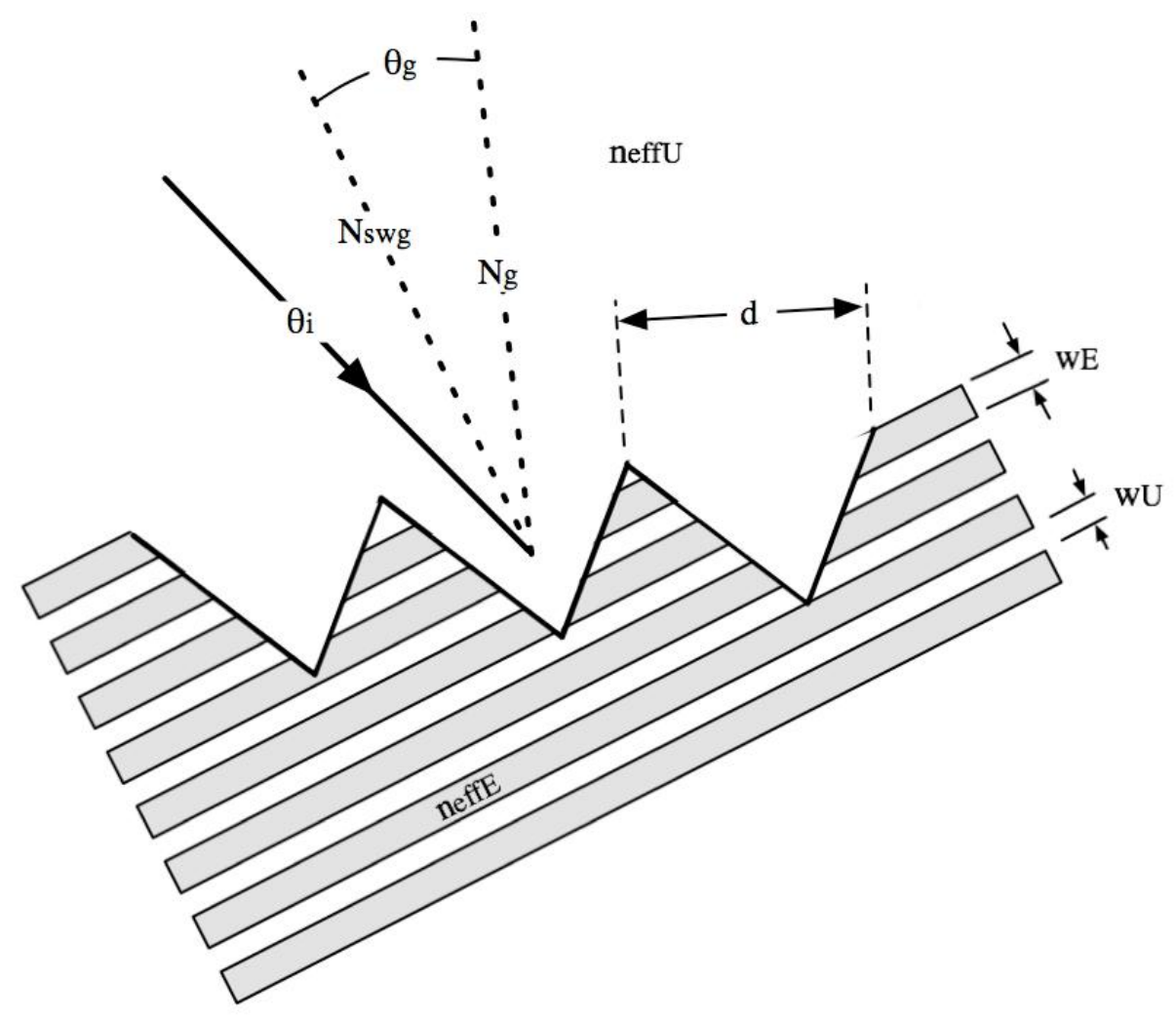

Fig. 4. Layout of the distributed grating with an arbitrary angle between the diffraction grating and Bragg structure.

Using scalar diffraction and the grating equation, the period $\mathrm{d}$, the working order $\mathrm{m}$, incidence angle $\theta_{\mathrm{i}}$ and diffraction $\theta_{\mathrm{d}}$ angles for the grating are determined. We then assume the Littrow (auto-collimation) incidence configuration, i.e. $\theta_{\mathrm{i}}=-$ $\theta_{\mathrm{d}}$, which yields:

$$
\sin \theta_{i}=\frac{-m \lambda_{0}}{2 n_{\text {eff }} d}
$$

where $\lambda_{0}$ is the central free-space wavelength and $\mathrm{n}_{\text {effu }}$ is the effective mode index in the (un-etched) slab waveguide. The scalar design provides initial values for the incidence angle, working order and, resolving power and angular dispersion of the grating. The second step consists of the selection of a material system and the design of the input and slab waveguide portions of the structure. A $260 \mathrm{~nm}$ silicon over a $1000 \mathrm{~nm} \mathrm{SiO}_{2}$ cladding material was chosen. The width and taper properties of the input waveguides were modeled using a three-dimensional beam propagation method (BPM), as mentioned previously. Effective indices for the etched and un-etched regions of the device were extracted from the BPM model. With the scalar grating and input waveguides designs completed, multilayer grating theory is used to design the subwavelength portions of the structure. The subwavelength grating geometry is tuned using the bisecting angle between the incident and diffracted working orders of the grating:

$$
w_{E, U}=\frac{\lambda_{0}}{4 n_{e f E, U} \cos \left(\theta_{g}-\theta_{i}\right)}
$$

with $\theta_{\mathrm{g}}$ defined as the angle between the grating normal and the subwavelength grating normal and where $\mathrm{w}_{\mathrm{E}, \mathrm{U}}$ and $\mathrm{n}_{\mathrm{effE}, \mathrm{U}}$ are the widths and effective indices of the etched and un-etched portions of the subwavelength grating, respectively. The subwavelength grating period is then $d_{\text {swg }}=w_{E}+w_{U}$ and the subwavelength duty factor $\alpha=w_{E} /\left(d_{\text {swg }}\right)$. The resulting 
design values were then used in a two-dimensional RCWA to model the behavior of the distributed etched grating in order to optimize the blaze properties for one grating period. Diffraction efficiencies as well as field profiles are extracted from the RCWA and a far-field transformation is applied to obtain the intensity profile at the output waveguide location and coupling efficiencies to the output waveguides, if present. An FDTD model was also used to verify the results of the RCWA. The output waveguides are positioned along the Rowland circle at the appropriate position for each of the output wavelengths.

Two possible working order configurations have been explored: the echelle and echelette structures. The model predicts a total bandwidth of approximately $118 \mathrm{~nm}$ and $60 \mathrm{~nm}$ for the echelette and echelle designs, respectively. The echellette design can demultiplex 147 100-GHz channels with a chip size of $27 \times 5 \mathrm{~mm}$, and the echelle design can demultiplex 37 $100-\mathrm{GHz}$ channels with a chip size of $4 \times 1 \mathrm{~mm}$. The working order was $\mathrm{m}=-10$ for both structures. The diffraction efficiencies obtained with RCWA were $98 \%$ and $79 \%$ for the echelette and echelle designs, respectively.

Due to fabrication constraints, we chose to fabricate a distributed grating echelle test structure, as a proof of concept for this design. The test echelle structure layout is shown in Fig. 5.

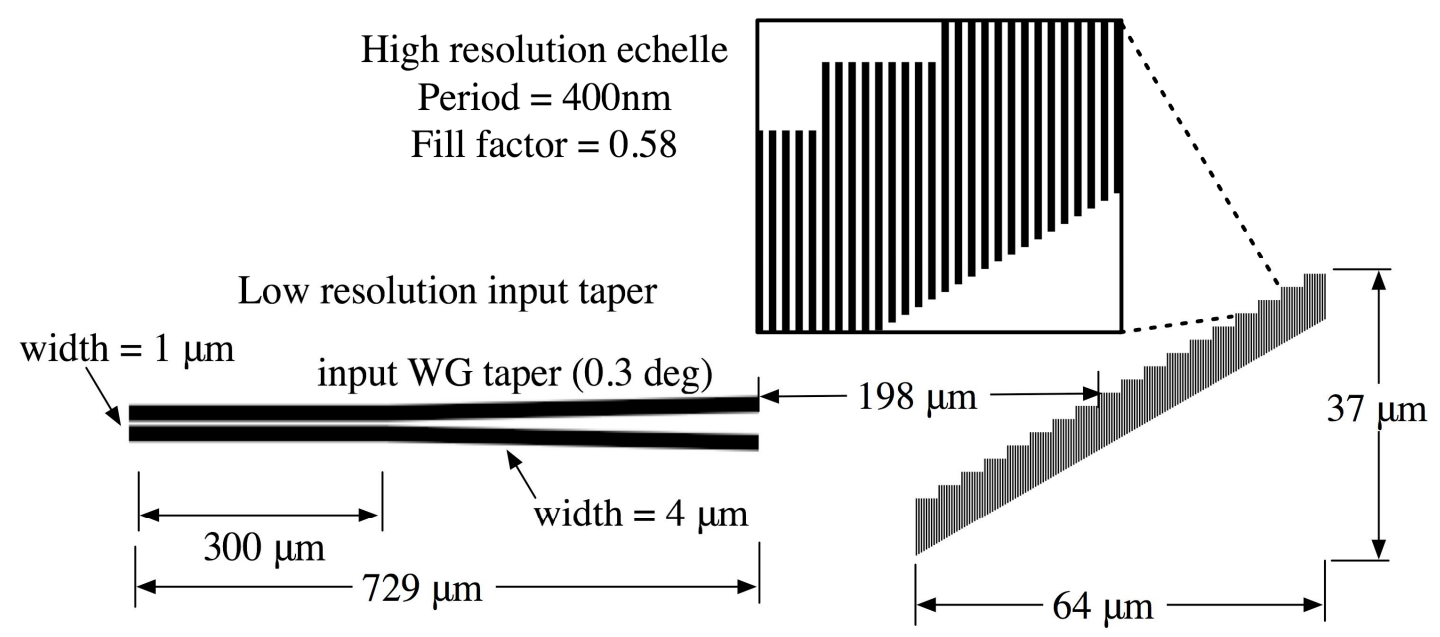

Fig. 5 Test Structure Layout.

For the proof of concept device, a single polarization state was chosen, specifically the TM mode of the input waveguide, corresponding to the TE mode for the distributed grating. The predicted on-chip efficiency of the distributed EDG from RCWA is $79 \%$ and this value is confirmed by FDTD. The far field energy distribution from the test structure is shown in Fig. 6. 


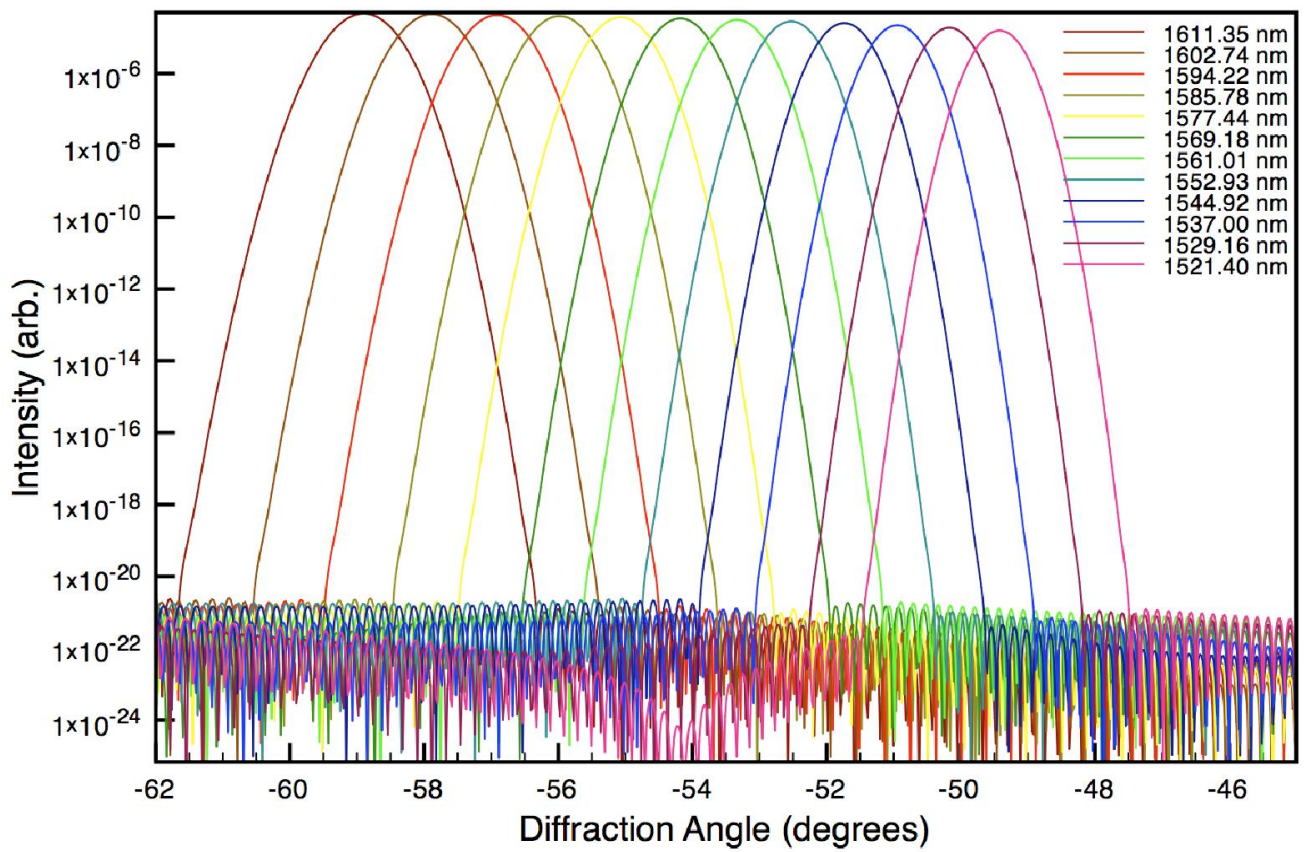

Fig. 6. Performance of the distributed echelle test structure, from FDTD.

\section{DEVICE FABRICATION}

The distributed EDG is fabricated using $20 \mathrm{kV}$ electron-beam lithography (E-beam) pattern writing and single step electron cyclotron resonance (ECR)-etching with $\mathrm{Cl}_{2}$ gas at $0^{\circ} \mathrm{C}$ chamber temperature and $200 \mathrm{~W}$ RF power. A $60 \mathrm{~nm} \pm 5 \mathrm{~nm}$ etch depth was realized for the Bragg portion of the structure using this fabrication technique. The result is shown in Fig. 7. 


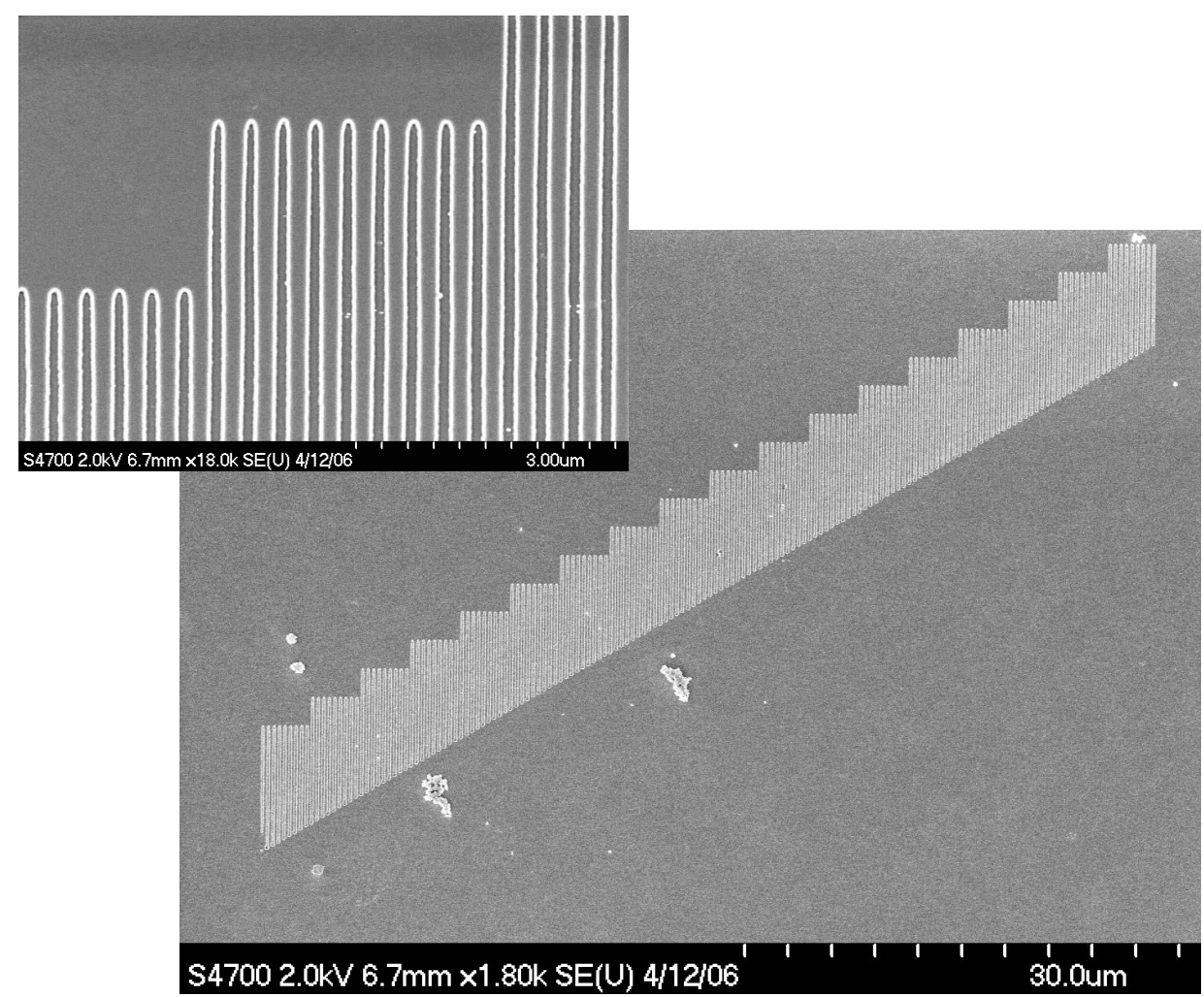

Fig. 7. Fabricated Echelle, showing distributed grating (inset).

Using an AFM the etch depth for the distributed grating was characterized. The achieved etch depth was measured at $61 \mathrm{~nm} \pm 3 \mathrm{~nm}$. The etch depth uniformity across different grooves of the echelle profile was measured to be $\pm 5 \mathrm{~nm}$. The measured period of the device was $394 \mathrm{~nm} \pm 2 \mathrm{~nm}$. The width of the etched region was measured to be $150 \mathrm{~nm}$. This is a large deviation from the target value and is attributed to incorrect exposure dosage from the e-beam patterning. Modeling shows that this error in fabrication will shift the reflection spectrum of the subwavelength grating towards longer wavelengths, particularly reducing the efficiency of the $1480-1520 \mathrm{~nm}$ band. At $1500 \mathrm{~nm}$ the expected reflection efficiency is expected to be reduced from $97 \%$ to $70 \%$. The etch profile for the structure is shown in Fig. 8 . 


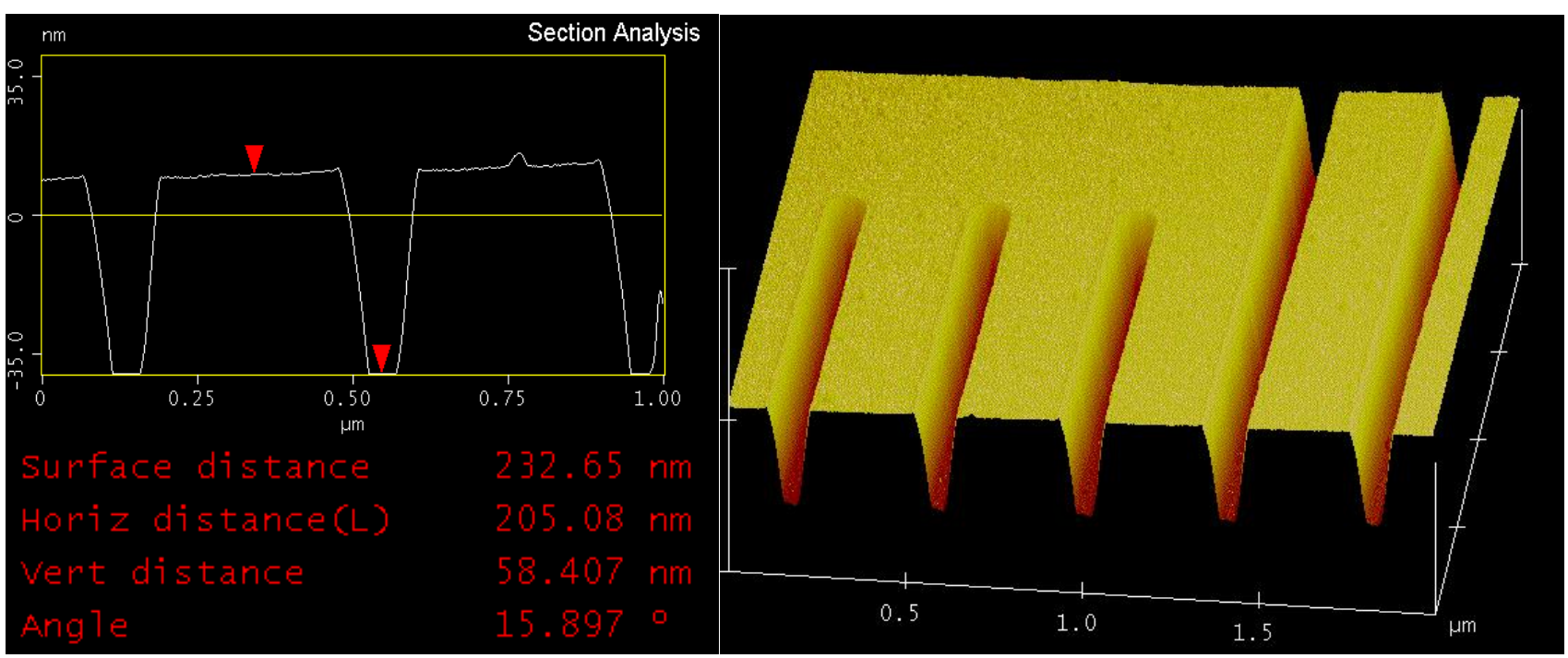

Fig. 8. Depth characterization of the sample using an atomic force microscope. The measured depth is $60 \pm 5 \mathrm{~nm}$.

\section{DEVICE CHARACTERIZATION}

Light was injected into the input waveguide using a tapered optical fiber. Top field scattering was observed using a sensitive InGaAs camera, as shown in Fig. 9. A 3.9dB overall increase in reflection was measured when sending light to the distributed structure rather than sending it into a neighboring waveguide.

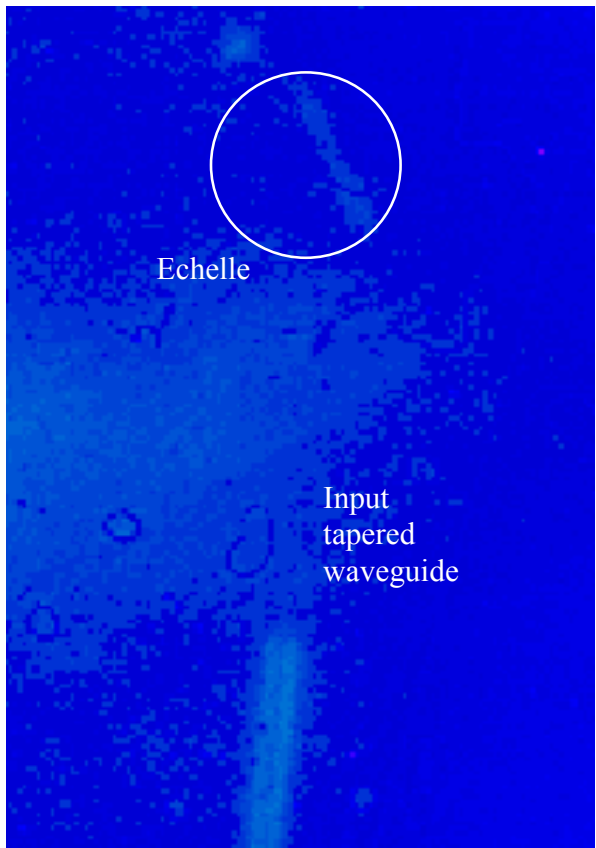

(a)

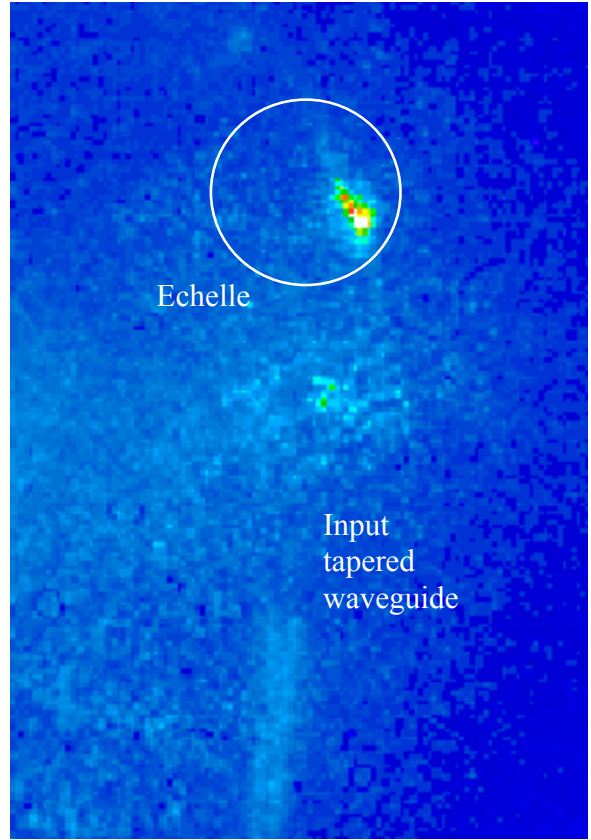

(b)

Fig. 9. Optical testing: a) structure only, b) light impinging on the distributed echelle 
Optical characterization of the test structure was performed by measuring the retro-diffracted light from the grating. The result of this measurement is shown in Fig. 10.

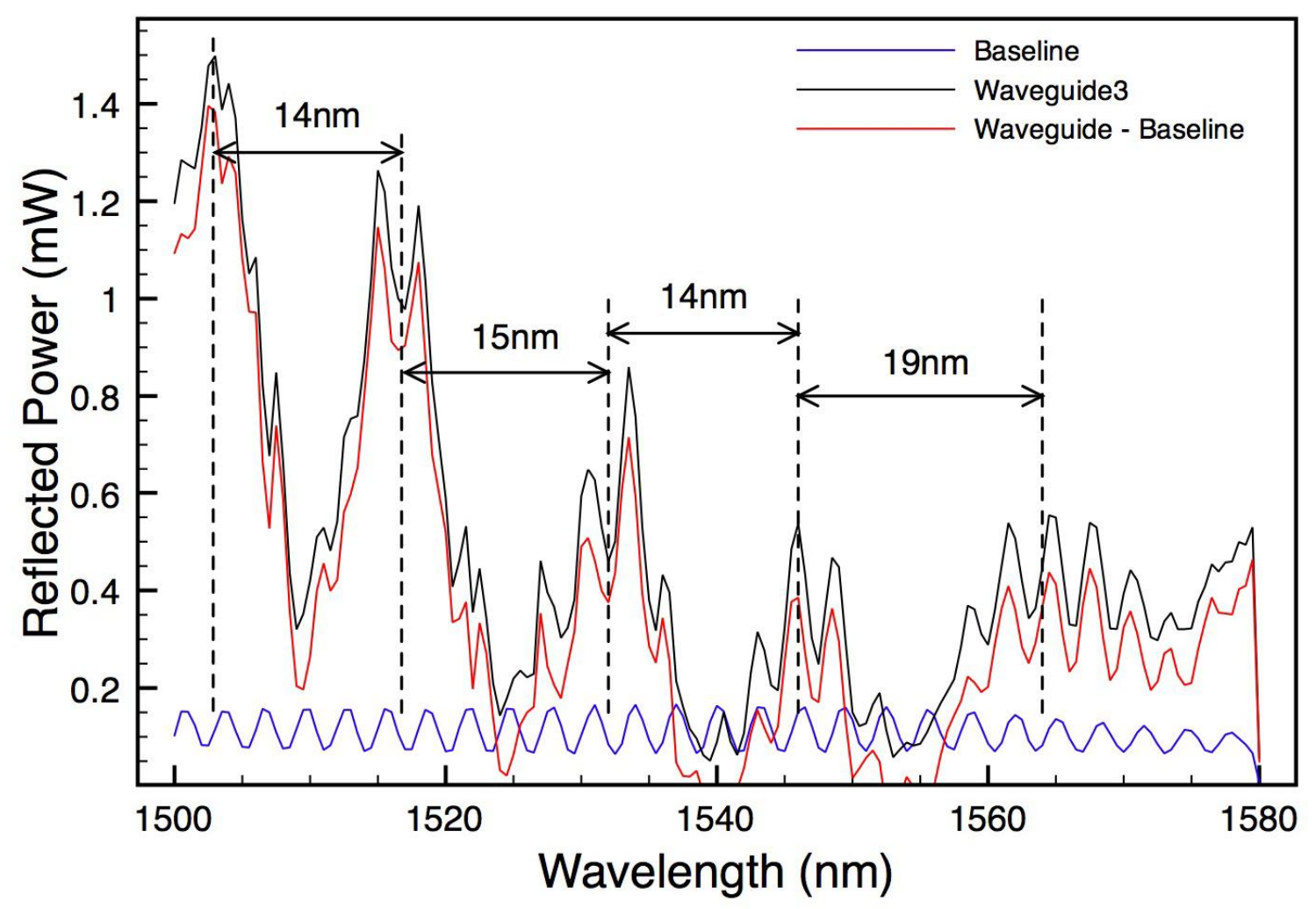

Fig. 10. Optical measurement showing retro-reflected light intensity for the structure.

The main peak separation of $15 \mathrm{~nm}$ is consistent with theoretical predictions, resulting from the diffraction over the illuminated grating width of $37 \mu \mathrm{m}$. This corresponds to the expected resolution of the test grating, which is mainly determined by the number of periods illuminated. The fast oscillations $(3 \mathrm{~nm})$ are consistent with features larger than the grating $(163 \mu \mathrm{m})$, corresponding either to the separation of the input taper to the grating $(198 \mathrm{~nm})$ or the straight portion of the input waveguide $(300 \mathrm{~nm})$. The determination of the source of the small oscillations is limited by the wavelength resolution of the measurement $(1 \mathrm{~nm})$.

\section{CONCLUSION}

A distributed-grating device in SOI was proposed as a novel approach to the etched grating demultiplexer. Low (echelette) and high (echelle) working order configurations were designed. The echelle grating yields a higher spectral resolution $(\sim 0.2 \mathrm{deg} / \mathrm{nm})$ and smaller device size at the cost of reduced bandwidth $(\sim 60 \mathrm{~nm})$ and diffraction efficiency $(79 \%)$. A test echelle structure was designed, modeled and fabricated. Spectral measurements on a proof-of-concept structure show promising results. 


\section{ACKNOWLEDGEMENTS}

This work was supported by the Agile All-Photonic Networks (NSERC). The authors would like to thank Andras G. Pattantyus-Abraham from UBC for advice on etching. The authors would also like to acknowledge the Western Canada Research Grid computation facilities and Lumerical FDTD.

\section{REFERENCES}

1. E. S. Koteles, and J. J. He, "Waveguide grating-based spectrometric transducers," IEEE Instrumentation and Measurement Magazine 7, 33 (2004).N. Bluzer and A. S. Jensen, "Current readout of infrared detectors," Opt. Eng. 26(3), 241-248 (1987).

2. H. Jian-Jun, et al., "Monolithic integrated wavelength demultiplexer based on a waveguide Rowland circle grating in InGaAsP/lnP," Journal of Lightwave Technology 16, 631 (1998).

3. E. Bisaillon, et al., "A Shallow-Etched Multilayer Grating-based Wavelength Demultiplexer in SOI", in IEEELEOS Annual Meeting Proceedings, 596 (2005).

4. H. Wei, and L. Li, "All-dielectric reflection gratings: A study of the physical mechanism for achieving high efficiency," Applied Optics 42, 6255 (2003).

5. M. Neviere, and E. Popov, Light Propagation in Periodic Media, Differential Theory and Design (Marcel Dekker Inc., New York, 2004). 